\title{
Outflow changes in positive provocative tests
}

\author{
R. MAPSTONE \\ From St. Paul's Eye Hospital, Liverpool
}

SUMMARY The outflow changes in 26 eyes were measured during the course of a positive provocative test with pilocarpine and phenylephrine. Three separate patterns of response occurred: (1) One dose of pilocarpine and phenylephrine produced an acute attack with a large reduction in outflow. (2) The first dose of pilocarpine and phenylephrine produced a fall in pressure and increase in outflow. The second dose reversed this effect and closed the angle. (3) The first dose of pilocarpine and phenylephrine increased pressure by an insignificant amount and decreased outflow. The second dose accentuated this response and produced an acute attack.

The effect of pilocarpine and phenylephrine drops instilled simultaneously into eyes at risk of developing closed-angle glaucoma is to produce a significant pressure increase (greater than $8 \mathrm{mmHg}$ ) in 60 to $70 \%$ (Mapstone, 1976). The purpose of this paper is to describe the changes in outflow facility that occur during a positive provocative test.

\section{Material and methods}

The provocative test is conducted as follows: At zero hours the intraocular pressure was recorded, outflow facility measured and pilocarpine drops $2 \%$ plus phenylephrine drops $10 \%$ were instilled. Subsequently at approximately half-hourly intervals, phenylephrine $10 \%$ was instilled and the intraocular pressure recorded. As soon as pressure had increased significantly (greater than $8 \mathrm{mmHg}$ ) the outflow facility was measured and the test terminated by thymoxamine drops $\frac{1}{2} \%$ and intravenous acetazolamide $500 \mathrm{mg}$.

If after the lapse of $1 \frac{1}{2}$ hours from the start there had been no significant increase in pressure, the pressure was recorded, facility of outflow measured, and a further dose of pilocarpine drops $2 \%$ and phenylephrine drops $10 \%$ instilled. One hour later (that is $2 \frac{1}{2}$ hours from the start of the test) if there was no significant increase in pressure the test was terminated by the instillation of thymoxamine drops $\frac{1}{2} \%$. If there was a significant increase in pressure the facility of outflow was measured and the test terminated with thymoxamine drops $\frac{1}{2} \%$ and intravenous acetazolamide $500 \mathrm{mg}$.

Address for reprints: Mr R. Mapstone, St. Paul's Eye Hospital, Old Hall Street, Liverpool L3 9PF
The material consists of 26 eyes from 26 patients who had closed-angle glaucoma in the contralateral eye and a positive provocative test. Negative test results are not described or discussed in this paper.

\section{Results}

Three different patterns of response occurred:

(1) Twelve of the 26 eyes developed a positive provocative test after 1 dose of pilocarpine and phenylephrine. The mean values are recorded in Fig. 1 and show that pressure increased from a mean of $16 \mathrm{mmHg}$ to a mean of $35.1 \mathrm{mmHg} 67$ minutes after the start of the test. At the same time the facility of outflow (C) decreased from a mean of 0.21 to a mean of 0.05 .

(2) In 7 eyes the first dose of pilocarpine and phenylephrine produced a fall in pressure and an increase in $\mathrm{C}$, whereas the second dose of pilocarpine
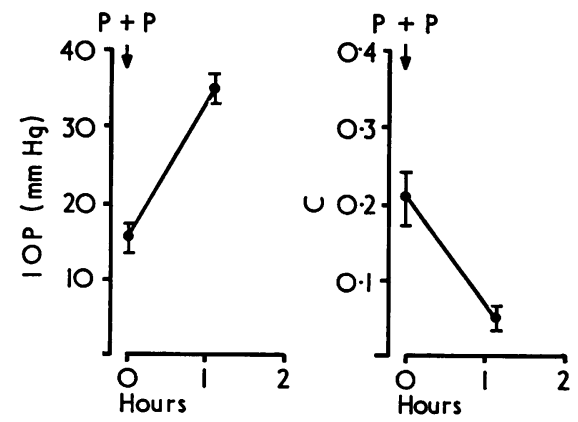

Fig. 1 Response of 12 eyes to provocative testing with pilocarpine and phenylephrine, one dose is sufficient to produce an acute attack. Mean values and standard error recorded. $P+P=$ Pilocarpine and Phenylephrine 
and phenylephrine produced an opposite response. Fig. 2 records the results and shows that after the first dose of pilocarpine and phenylephrine pressure fell from 19.7 to $16.4 \mathrm{mmHg}$ and $\mathrm{C}$ increased from $0 \cdot 17$ to $0.27 ; 55$ minutes after the second dose of pilocarpine and phenylephrine pressure increased from 16.4 to $32.7 \mathrm{mmHg}$ and $\mathrm{C}$ decreased from 0.27 to 0.02 .

(3) In the remaining 7 eyes the first dose of pilocarpine and phenylephrine produced an increase in pressure and a decrease in $\mathbf{C}$, changes which were accentuated by the second dose of pilocarpine and phenylephrine. Fig. 3 records the results and shows that after the first dose of pilocarpine and phenylephrine pressure increased from 14.9 to $16.6 \mathrm{mmHg}$ and $C$ decreased from 0.24 to $0.14 ; 74$ minutes after the second dose of pilocarpine and phenylephrine
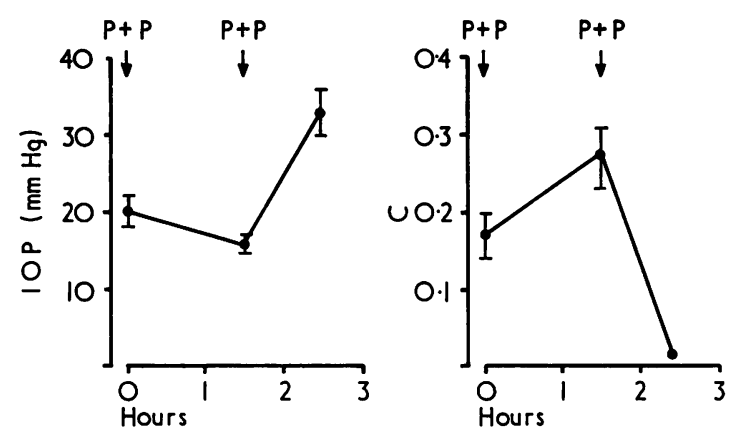

Fig. 2 Response of 7 eyes to provocative testing with pilocarpine and phenylephrine; the first dose decreases pressure and increases $C$. The second dose has an opposite effect. Mean values and standard error recorded. $P+P$ $=$ Pilocarpine and Phenylephrine
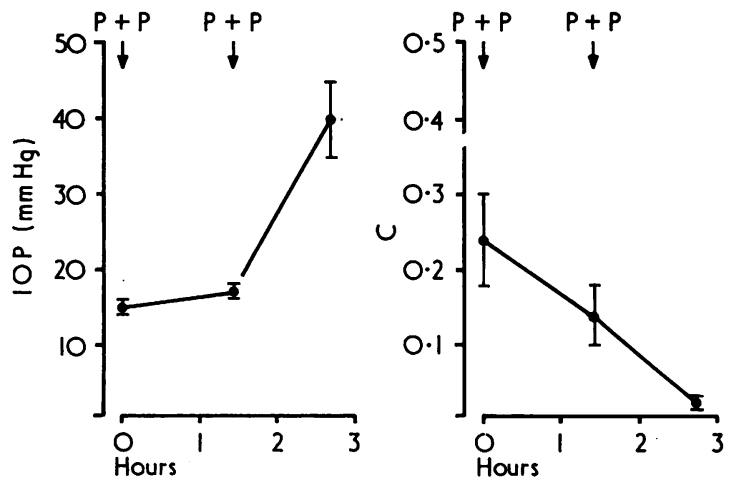

Fig. 3 Response of 7 eyes to provocative testing with pilocarpine and phenylephrine; the first dose increases pressure and decreases outflow, a change accentuated by the second dose. Mean values and standard error recorded. $P+P=$ Pilocarpine and Phenylephrine

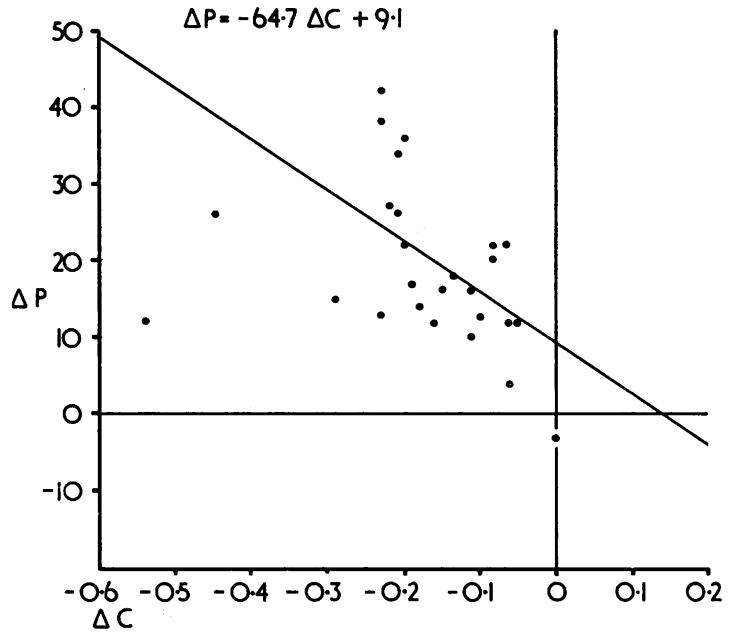

Fig. $4 A$ plot of the total change in pressure $(\Delta P)$ against the total change in $C(\Delta C)$ in the 26 eyes. The equation of the regression line is $\Delta P=-67 \cdot 4 \Delta C+9 \cdot 1$; the correlation coefficient is -0.6

pressure increased from 16.6 to $40 \mathrm{mmHg}$ and $\mathrm{C}$ decreased from $0 \cdot 14$ to $0 \cdot 02$.

A plot of the total change in pressure $(\Delta \mathrm{P})$ against the total change in outflow $(\Delta C)$ is shown in Fig. 4. The equation of the regression line is $\Delta \mathbf{P}=-67 \cdot 4 \Delta \mathrm{C}+9 \cdot 1$. The correlation coefficient is -0.6 , that is, there is a highly significant negative linear correlation between these two variables $(\mathrm{P}<0.001)$.

\section{Discussion}

Normal eyes respond to provocative testing with pilocarpine and phenylephrine by developing an increase in outflow (Mapstone, 1977). In the 26 eyes described here there was a very significant decrease in outflow at the end of the test. Since there is no reason to suppose that their outflow systems are in any way different from normal-apart from a high probability of developing angle closure-the most probable explanation of the reduction in $\mathrm{C}$ is angle closure. This conclusion is supported by the presence of a highly significant negative linear correlation between the changes in outflow and pressure.

The 26 eyes showed 3 separate patterns of response. The first (Fig. 1) would seem predictable-that is, approximately an hour after the start of the test the angle was closed by irido-corneal contact, and pressure increased by $20 \mathrm{mmHg}$. It is also apparent that the angle became closed as the pupil moved up to the position of mid-dilatation.

The 7 eyes exhibiting the second response (Fig. 2) 
increased $\mathrm{C}$ by $63 \%$ after the lapse of $1 \frac{1}{2}$ hours, and pressure decreased by an insignificant amountthat is, no portion of the angle closed as the pupil moved to mid-dilatation. The instillation of a second dose of pilocarpine and phenylephrine then produced an opposite response, with the angle occluding almost completely.

In the third response (Fig. 3) 80 minutes after the first dose of pilocarpine and phenylephrine $\mathrm{C}$ decreased by $55 \%$, but pressure had increased by an insignificant amount. At the same time the pupil had moved to mid-dilatation. It is, of course, possible that after an appropriate period this group of eyes would have proceeded inexorably to an acute attack even if no further drugs were instilled. In the event an additional dose of pilocarpine and phenylephrine was instilled, and 74 minutes later the angle was almost completely occluded and pressure increased by $23 \mathrm{mmHg}$. This group of eyes therefore produced partial angle closure as the pupil moved to mid-dilatation but an insufficient pressure increase to produce a positive result. The addition of pilocarpine and phenylephrine at middilatation then closed the angle.

The clinical implications of these varying patterns of response are explored in subsequent papers. It is, however, apparent that a substantial proportion of the angle can close without the production of a significant pressure change. The limiting factors are the extent and duration of angle closure.

I am grateful to colleagues who referred patients for study and to Mrs E. Tubb for secretarial help.

\section{References}

Mapstone, R. (1976). British Journal of Ophthalmology, 60, 115.

Mapstone, R. (1977). British Journal of Ophthalmology, 61, 510. 